An asteroseismic constraint on the mass of the axion from the period drift of the pulsating DA white dwarf star L19-2

This content has been downloaded from IOPscience. Please scroll down to see the full text. JCAP07(2016)036

(http://iopscience.iop.org/1475-7516/2016/07/036)

View the table of contents for this issue, or go to the journal homepage for more

Download details:

IP Address: 130.183.82.44

This content was downloaded on 04/09/2016 at 20:10

Please note that terms and conditions apply.

You may also be interested in:

Interaction of axions with relativistic spinning particles

V A Popov and A B Balakin

Spectroscopic and photometric study of Kepler asteroseismic targets

Joanna Molenda-akowicz, Antonio Frasca, David Latham et al.

PROPERTIES OF 42 SOLAR-TYPE KEPLER TARGETS FROM THE ASTEROSEISMIC MODELING PORTAL T. S. Metcalfe, O. L. Creevey, G. Doan et al.

SUB-INERTIAL GRAVITY MODES IN THE B8V STAR KIC 7760680 REVEAL MODERATE CORE DEIERTIAAOOTHINGSAME MOXNG

Ehsan Moravveji, Richard H. D. Townsend, Conny Aerts et al.

Revisiting the axion bounds from the Galactic white dwarf luminosity function

M.M. Miller Bertolami, B.E. Melendez, L.G. Althaus et al.

g-MODES IN THE RAPIDLY ROTATING Idelta SCUTI STAR RASALHAGUE

J. D. Monnier, R. H. D. Townsend, X. Che et al.

ASTEROSEISMIC AGE AND RADIUS OF KIC 11026764

T. S. Metcalfe, M. J. P. F. G. Monteiro, M. J. Thompson et al. 


\section{An asteroseismic constraint on the mass of the axion from the period drift of the pulsating DA white dwarf star L19-2}

Alejandro H. Córsico, ${ }^{a, b}$ Alejandra D. Romero, ${ }^{c}$ Leandro G. Althaus, ${ }^{a, b}$ Enrique García-Berro, ${ }^{d, e}$ Jordi Isern, ${ }^{e, f}$ S.O. Kepler, ${ }^{c}$ Marcelo M. Miller Bertolami, ${ }^{a, b}$ Denis J. Sullivan ${ }^{g}$ and Paul Chote ${ }^{g, h}$

\footnotetext{
${ }^{a}$ Grupo de Evolución Estelar y Pulsaciones, Facultad de Ciencias Astronómicas y Geofísicas, Universidad Nacional de La Plata, Paseo del Bosque s/n, (1900) La Plata, Argentina

${ }^{b}$ Instituto de Astrofísica La Plata, CONICET-UNLP,

Paseo del Bosque s/n, (1900) La Plata, Argentina

${ }^{c}$ Departamento de Astronomia, Universidade Federal do Rio Grande do Sul, Av. Bento Goncalves 9500, Porto Alegre 91501-970, RS, Brazil

${ }^{d}$ Departament de Física Aplicada, Universitat Politècnica de Catalunya,

c/Esteve Terrades, 5, 08860 Castelldefels, Spain

${ }^{e}$ Institute for Space Studies of Catalonia, IEEC,

c/Gran Capità 2-4, Edif. Nexus 104, 08034 Barcelona, Spain

${ }^{f}$ Institut de Ciències de l'Espai, CSIC, Campus UAB, Facultat de Ciències, Torre C-5, 08193 Bellaterra, Spain

${ }^{g}$ School of Chemical \& Physical Sciences, Victoria University of Wellington, P.O. Box 600, Wellington 6140, New Zealand

${ }^{h}$ Department of Physics, University of Warwick, Coventry CV4 7AL, U.K.

E-mail: acorsico@fcaglp.unlp.edu.ar, alejandra.romero@ufrgs.br, althaus@fcaglp.unlp.edu.ar, enrique.garcia-berro@upc.edu, isern@ieec.cat, kepler@if.ufrgs.br, mmiller@fcaglp.unlp.edu.ar, denis.sullivan@vuw.ac.nz, p.chote@warwick.ac.uk
}

Received May 23, 2016

Revised July 1, 2016

Accepted July 12, 2016

Published July 21, 2016 
Abstract. We employ an asteroseismic model of L19-2, a relatively massive $\left(M_{\star} \sim 0.75 M_{\odot}\right)$ and hot $\left(T_{\text {eff }} \sim 12100 \mathrm{~K}\right.$ ) pulsating DA (H-rich atmosphere) white dwarf star (DAV or ZZ Ceti variable), and use the observed values of the temporal rates of period change of its dominant pulsation modes $(\Pi \sim 113 \mathrm{~s}$ and $\Pi \sim 192 \mathrm{~s})$, to derive a new constraint on the mass of the axion, the hypothetical non-barionic particle considered as a possible component of the dark matter of the Universe. If the asteroseismic model employed is an accurate representation of L19-2, then our results indicate hints of extra cooling in this star, compatible with emission of axions of mass $m_{\mathrm{a}} \cos ^{2} \beta \lesssim 25 \mathrm{meV}$ or an axion-electron coupling constant of $g_{\text {ae }} \lesssim 7 \times 10^{-13}$.

Keywords: axions, stars, white and brown dwarfs

ArXiv ePrint: 1605.06458 


\section{Contents}

1 Introduction and context 1

2 Measurements of $\dot{\Pi}$ for the periods at $113 \mathrm{~s}$ and $192 \mathrm{~s} \quad 4$

3 Asteroseismic model for L19-2 5

3.1 Mode trapping 6

3.2 Uncertainties in the theoretical value of $\dot{\Pi} \quad 8$

4 Impact of axion emission on the rates of period change 10

$\begin{array}{lll}5 & \text { The axion mass } & 10\end{array}$

6 Discussion and conclusions 11

\section{Introduction and context}

Charge-parity (CP) symmetry [1,2] states that the laws of physics should be the same if particles were switched with their antiparticles (C symmetry) and their spatial coordinates were inverted ( $\mathrm{P}$ symmetry). The theory of quantum chromodynamics (QCD) predicts that the $\mathrm{CP}$ symmetry must be broken in strong interactions. However, this violation is not observed in nature, at variance with what happens in the electro-weak theory [3]. This constitutes a long-standing problem in particle physics, known as the "strong CP" problem [4].

A possible solution to strong CP problem was proposed about 40 years ago by postulating the existence of a new particle, the pseudo Nambu-Goldstone boson - that is, a boson with a small mass [5] - known as an axion [6-8]. At present, however, this particle has not been detected. Currently, axions are the target of many theoretical and experimental studies aimed at proving their existence. One the main interests driving these experiments is that axions are candidates for being one of the possible components of cold dark matter (CDM). The contribution of axions to CDM, however, depends on their mass [9], that is a free parameter of the theory that postulates their existence. Axions are neutral (they have no electric charge), and they interact very weakly with normal matter and radiation. Among the various types of axion models, the most commonly discussed in the literature are the KVSZ model $[10,11]$, where the axions couple with photons and hadrons, and the DFSZ model $[12,13]$, where they also couple to charged leptons, like electrons. In this work, we focus on DFSZ axions, that is those that interact with electrons.

The coupling strength of DFSZ axions to electrons is defined through a dimensionless coupling constant, $g_{\mathrm{ae}}$, which is related to the mass of the axion, $m_{\mathrm{a}}$, through the equation [5]:

$$
g_{\mathrm{ae}}=2.8 \times 10^{-14} \frac{m_{\mathrm{a}} \cos ^{2} \beta}{1 \mathrm{meV}},
$$

where $\cos ^{2} \beta$ is a free, model-dependent, parameter that is usually set equal to unity. Since the theory does not place any constraint on the mass of axions, it must be inferred from either sophisticated terrestrial experiments [14], or indirectly by using well-studied properties of stars [5]. In this paper, we use white dwarf stars to constrain the mass of the axion. 
White dwarfs are the very late evolutionary state of most low- and intermediate-mass stars $\left(M_{\star} \sim 0.8-10 M_{\odot}\right)$, including our Sun [15]. Because in the degenerate core of white dwarfs free electrons are abundant, axions would be copiously produced in their interiors [16]. Moreover, because white dwarfs are strongly degenerate and do not have relevant nuclear energy sources, their evolution consists in a slow cooling process in which the gravothermal energy release is the main energy source driving their evolution [17]. Thus, any additional cooling mechanism should be prominent, and have observable consequences. At the typical temperatures and densities $(\log T \sim 6, \log \rho \sim 6)$ found in the cores of white dwarfs, the emission of DFSZ axions would take place in the deepest regions of these stars through Compton, pair annihilation, and bremmsstrahlung processes, this last mechanism being the dominant one [16]. Under these conditions, the axion emission rate is given by $[18,19]$ :

$$
\epsilon_{\mathrm{a}}=1.08 \times 10^{23} \frac{g_{\mathrm{ae}}^{2}}{4 \pi} \frac{Z^{2}}{A} T_{7}^{4} F(T, \rho) \quad[\mathrm{erg} / \mathrm{g} / \mathrm{s}] .
$$

where $Z$ and $A$ are the atomic and mass numbers, respectively, $T_{7}$ is the temperature in units of $10^{7} \mathrm{~K}$, and $F=F(T, \rho)$ is a function of the density and temperature that takes into account the Coulomb plasma effects. As can be seen, the axion emission is determined by the coupling strength. In turn, the strength of the coupling of axions with electrons depends on the axion mass - see eq. (1.1). Consequently, the more massive the axions are, the larger the axion emission is. Since axions can freely escape from the interior of white dwarfs, their emission would accelerate cooling, with more massive axions producing larger cooling rates.

One of the possible methods to determine the mass of the axion consists in using the white dwarf luminosity function. The luminosity function of white dwarfs is defined as the number of white dwarfs per unit bolometric magnitude and unit volume. Clearly the cooling rate of white dwarfs will have an impact on the form of the luminosity function. It was found [20] some time ago that when axion emissivity is included in the white dwarf evolutionary models, the agreement between the theoretical calculations and the observed white dwarf luminosity function significantly improves $[20,21]$. These early computations were enhanced later on by employing new white dwarf luminosity functions of the Galactic Disk derived from different catalogues, and adopting new theoretical cooling sequences constructed on the basis of full evolutionary white dwarfs models that incorporate self-consistently the effects of axions on the thermal structure of the white dwarf [22]. This later study found the need for extra cooling emission from white dwarfs to be marginal and dependent on the derivation of the white dwarf luminosity function.

The second method of interest used to measure the mass of the axion involves using the rate of period change of pulsating white dwarfs. Pulsating white dwarfs with H-rich envelopes, also called ZZ Ceti or DAV variable stars, constitute the most numerous class of degenerate pulsators, with over 160 members known to date [23]. ZZ Ceti stars exhibit multiperiodic luminosity changes resulting from spheroidal modes - spheroidal modes satisfy $(\vec{\nabla} \times \vec{\xi})_{r}=0$, where $\vec{\xi}$ is the Lagrangian displacement vector [24]. The modes are non-radial gravity-mode $(g$-mode) pulsations of low degree $(\ell \leq 2)$ with periods ranging from 70 to $1500 \mathrm{~s}[15,25,26]$. The pulsation periods $(\Pi)$ of ZZ Ceti stars experience a secular drift as the stars cool, allowing for measurable rates of period change, $\dot{\Pi} \equiv d \Pi / d t$. Indeed, as the temperature in the core of a white dwarf decreases, the plasma increases its degree of degeneracy so the Brunt-Väisälä (buoyancy) frequency - the critical frequency of $g$-mode pulsations [24] - decreases, and the pulsational spectrum of the star is shifted to longer periods. On the other hand, residual gravitational contraction (if present) acts in the opposite 
direction, thus shortening the pulsation periods. The competition between the increasing degeneracy and gravitational contraction gives rise to a detectable $\dot{\Pi}$. In particular, it has been shown [27] that the rate of change of the pulsation periods is related to the rates of change of the temperature at the region of the period formation, $\dot{T}$, and of the stellar radius, $\dot{R}_{\star}$, according to the following order-of-magnitude expression:

$$
\frac{\dot{\Pi}}{\Pi} \approx-a \frac{\dot{T}}{T}+b \frac{\dot{R_{\star}}}{R_{\star}}
$$

where $a$ and $b$ are constants whose values depend on the details of the white dwarf modeling (however, both $a$ and $b \approx 1$ ). The first term in eq. (1.3) corresponds to the rate of change in period induced by the cooling of the white dwarf, and since $\dot{T}<0$, it is a positive contribution. The second term represents the rate of change due to gravitational contraction $(\dot{R}<0)$, and it is a negative contribution. In principle, the rate of change of the period can be measured by observing a pulsating white dwarf over a long time interval when one or more very stable pulsation periods are present in their light curves. In the case of pulsating DA white dwarfs, cooling dominates over gravitational contraction, in such a way that the second term in eq. (1.3) is usually negligible, and only positive values of the observed rate of change of period are expected.

The possibility of employing the measured rate of period change in the ZZ Ceti star G117-B15A to derive a constraint on the mass of axions was raised for the first time by [28]. G117-B15A ( $\left.T_{\text {eff }}=11430-12500 \mathrm{~K}, \log g=7.72-8.03,[29-33]\right)$ is the best studied star of the ZZ Ceti class, with pulsation periods at $215.20 \mathrm{~s}, 270.46 \mathrm{~s}$ and $304.05 \mathrm{~s}$ [34]. The evolution of DA white dwarfs with and without axion emission was considered [28], and the theoretical values of $\dot{\Pi}$ associated with the largest-amplitude mode with period at $\sim 215 \mathrm{~s}$ for increasing masses of the axion were compared to the observed rate of change of period with time of G117-B15A. Employing a semi-analytical treatment, $m_{\mathrm{a}} \cos \beta=8.7 \mathrm{meV}\left(g_{\mathrm{ae}}=2.4 \times 10^{-13}\right)$ was obtained [28]. Later, a detailed asteroseismic model for G117-B15A was computed [35], and $m_{\mathrm{a}} \cos \beta<4.4 \mathrm{meV}\left(g_{\mathrm{ae}}<1.2 \times 10^{-13}\right)$ was obtained. Subsequently, an upper limit of $26.5 \mathrm{meV}\left(g_{\mathrm{ae}}<7.4 \times 10^{-13}\right)$ was inferred for the axion mass using an improved asteroseismic model for G117-B15A, and a better treatment of the uncertainties involved [36]. Finally, employing the latest asteroseismic model [37] and the most recent determination of the rate of change of the period at $215 \mathrm{~s}$ of G117-B15A, $\dot{\Pi}=(4.19 \pm 0.73) \times 10^{-15} \mathrm{~s} / \mathrm{s}$ [38], the issue was revisited [39]. The $215 \mathrm{~s}$ period in the asteroseismic model of G117-B15A was associated with a $g$ mode trapped in the $\mathrm{H}$ envelope. The comparison of the theoretical rate of period change associated with the asteroseismic model with the observed one suggested the existence of an additional cooling mechanism in this pulsating white dwarf, consistent with axions of mass $m_{\mathrm{a}} \cos \beta=17.4 \mathrm{meV}\left(g_{\mathrm{ae}}=4.9 \times 10^{-13}\right)$.

R548, that is, ZZ Ceti itself (the prototype of the class), is another DAV star that has been intensively studied for the last few decades. Since the discovery of R548, there have been multiple attempts to measure the drift rate of its pulsation period at $\sim 213.13 \mathrm{~s}$, but only very recently the rate of change of this period with time has been measured for the very first time. Specifically, using 41 years of time-series photometry from 1970 to 2011 a value of $\dot{\Pi}=(3.3 \pm 1.1) \times 10^{-15} \mathrm{~s} / \mathrm{s}$ was obtained [40]. Using the measured rate of period change of R548 and an asteroseismic model for this star [37], a value of $m_{\mathrm{a}} \cos \beta=17.1 \mathrm{meV}$ $\left(g_{\mathrm{ae}}=4.8 \times 10^{-13}\right)$ was inferred [41]. The fact that both inferences for the axion mass are so similar is not surprising, in view of that both G117-B15A and R548 have similar effective temperatures, masses, and pulsation characteristics. In particular, it turns out that the 
$k=2, \ell=1$ mode lies at nearly identical periods for these stars $-\sim 215 \mathrm{~s}$ for G117-B15A and $\sim 213 \mathrm{~s}$ for R548.

Nevertheless, a cautionary remark is in order here. In particular, it turns out that the upper limit for the mass of the axion obtained using the white dwarf luminosity function of the Galactic Disk [22] is in conflict with a value as high as $m_{\mathrm{a}} \cos \beta \sim 17 \mathrm{meV}$, the value derived from emplopying the pulsating stars G117-B15A and R548. Indeed, a detailed fit of the shape of the white dwarf luminosity function of the Galactic Disk at the luminosities relevant for axion emission, disfavor axion masses $m_{\mathrm{a}} \cos \beta \gtrsim 10 \mathrm{meV}$. However, it is worth noting that the high mass of the axion derived using asteroseismic tools is a direct consequence of the identification of the $\sim 215 \mathrm{~s}(\sim 213 \mathrm{~s})$ mode of G117-B15A (R548) as a mode trapped in the $\mathrm{H}$ envelope $[39,41]$. The rate of period change of a trapped mode is reduced by residual gravitational contraction, because the oscillations of trapped modes are confined close to the surface [42]. Thus, it is of the utmost importance to study a pulsating white dwarf star in which the mode of interest (one for which the rate of period change is measured) is not a mode trapped in the envelope, but rather a mode with appreciable amplitude throughout the star.

Recently, it has been possible to derive for the first time a value of the rate of change for two periods in a third ZZ Ceti star, L19-2 [43]. This star (also known as MY Aps or WD1425-811) is a relatively bright pulsator, with a spectroscopic determination of its effective temperature and gravity of $T_{\text {eff }}=12100 \pm 200 \mathrm{~K}$ and $\log g=8.21 \pm 0.10$, respectively $[30,33]$. This star shows oscillation periods $\Pi$ (and milli-modulation ${ }^{1}$ amplitudes $A$ ) of $113.8 \mathrm{~s}(2.05 \mathrm{mma}), 118.7 \mathrm{~s}$ (1.34 mma), $143.6 \mathrm{~s}$ (0.49 mma), $192.6 \mathrm{~s}(5.92 \mathrm{mma})$, and $350.2 \mathrm{~s}(0.77 \mathrm{mma})$, that correspond to genuine eigenmodes [44]. The stability of the periods of the modes at $192.6 \mathrm{~s}$ and $113.8 \mathrm{~s}$ (associated to the largest-amplitude modes) has been recently investigated [43]. L19-2 is a well studied star and has been the subject of several asteroseismic analyses [45-48], for which we have reliable determinations of its effective temperature and surface gravity. Moreover, the results of all the asteroseismic models agree with the spectroscopic inferences. Thus, this star provides an unique opportunity to study the role of axions in white dwarf cooling.

In this paper, we employ a detailed asteroseismic model for L19-2 which is an improved version of that derived in ref. [48] and use the measurement of the rate of change of the $113.8 \mathrm{~s}$ and 192.6 s periods to set new constraints on the mass of the axion. The paper is organized as follows. In section 2 we give a succinct account of the measurements of the rate of period change in L19-2, while in section 3 we briefly present our asteroseismic model for L19-2, and describe in detail some propagation properties of the pulsation modes. In this section we also briefly discuss the uncertainties involved in our analysis. Section 4 follows, where we describe the impact that the inclusion of the axion emissivity in the asteroseismic model has on the pulsation modes. Section 5 is devoted to place an improved constraint on the axion mass. Finally, in section 6 we summarize our findings and we present our concluding remarks.

\section{Measurements of $\dot{\Pi}$ for the periods at $113 \mathrm{~s}$ and $192 \mathrm{~s}$}

L19-2 was found to be a pulsating white dwarf of the ZZ Ceti class in 1977 [49]. Subsequently, it was monitored during nearly five years from the South African Astronomical Observatory (SAAO) $[50,51]$. It was found that the star pulsates with at least five periods at $\sim 350$, $\sim 192, \sim 143, \sim 118$ and $\sim 113$ s. Also, it was possible to study the stability of the

\footnotetext{
${ }^{1}$ One milli-modulation amplitude is the Fourier amplitude of a signal with a fractional intensity variation of 0.1 per cent.
} 
modes with periods $\sim 113$ and $\sim 192 \mathrm{~s}$, and upper limits to the rate of period change of $\sim 2 \times 10^{-14} \mathrm{~s} / \mathrm{s}$ were placed for both periods. The measurement of $\dot{\Pi}$ in a pulsating white dwarf is a challenging observational endeavour, since a large photometric data set with a very long observational time baseline (typically decades) is necessary. Fortunately, L19-2 was the target of a Whole Earth Telescope (WET) [52] campaign in 1995 [44] employing only three southern observatories. In addition, single-site observations covering the interval 1994 to 2013 have been obtained using the one metre telescope at the University of Canterbury Mt John Observatory (UCMJO). A comprehensive analysis of the 1995 WET data along with single site observations will be presented in a forthcoming publication [53], but a summary has already been presented [43]. The stability of the periods at $\sim 192 \mathrm{~s}$ and $\sim 113 \mathrm{~s}$ was investigated, and a rate of period change of $\dot{\Pi}=(4.0 \pm 0.6) \times 10^{-15} \mathrm{~s} / \mathrm{s}$ for both modes was found. By taking into account a contribution due to proper motion of the star [54] of $\sim 1.0 \times 10^{-15} \mathrm{~s} / \mathrm{s}[43]$, we obtain $\dot{\Pi}=(3.0 \pm 0.6) \times 10^{-15} \mathrm{~s} / \mathrm{s}$. This is the value of $\dot{\Pi}$ for the periods at $\sim 113 \mathrm{~s}$ and $\sim 192 \mathrm{~s}$ that we will adopt in our analysis.

\section{Asteroseismic model for L19-2}

Here, we describe briefly the asteroseismic model for L19-2, and refer the interested reader to a previous publication [48] for further details concerning the asteroseismic approach adopted. A detailed asteroseismic analysis of L19-2 using a grid of DA white dwarf evolutionary models characterized by chemical profiles for both the core and the envelope consistent with the evolutionary history of the progenitor stars, and covering a wide range of stellar masses, thicknesses of the hydrogen envelope and effective temperatures was carried out [48]. These models were computed employing the LPCODE evolutionary code [55]. The evolutionary calculations were carried out from the ZAMS, through the thermally-pulsing and mass-loss phases on the AGB, and finally to the domain of planetary nebulae and white dwarfs. The effective temperature, the stellar mass and the mass of the $\mathrm{H}$ envelope of our DA white dwarf models are within the intervals $14000 \gtrsim T_{\text {eff }} \gtrsim 9000 \mathrm{~K}, 0.525 \lesssim M_{\star} \lesssim 1.050 M_{\odot}$, and $-9.4 \lesssim \log \left(M_{\mathrm{H}} / M_{\star}\right) \lesssim-3.6$, respectively. The value of the upper limit of $M_{\mathrm{H}}$ is dependent on $M_{\star}$ and is given by the previous evolution of the progenitor star. For simplicity, the mass of He was kept fixed at the value predicted by the evolutionary computations for each sequence. Different treatments of the crystallization process were taken into account self-consistently in the evolution and pulsations of our DA white dwarf models [48].

In order to find an asteroseismic model for L19-2, the model that minimizes a quality function that measures the goodness of the fit of the theoretical $\left(\Pi^{t}\right)$ to the observed $\left(\Pi^{\circ}\right)$ periods was sought [48]. The theoretical periods were assessed by means of the adiabatic version of the LP-PUL pulsation code [56]. A single best-fit model with the characteristics shown in table 1 was found. This model is an improved version of the asteroseismic model computed previously for L19-2 [48]. We identified the modes $118.7 \mathrm{~s}(k=1, \ell=1), 192.6 \mathrm{~s}$ $(k=2, \ell=1), \Pi^{\circ}=113.8 \mathrm{~s}(k=2, \ell=2)$, and $143.4 \mathrm{~s}(k=3, \ell=2)$. Within the scope of our set of pulsation models, these were the only possible identifications. The second column of table 1 contains the $T_{\text {eff }}, \log g$ and $M_{\star}$ of L19-2 according to spectroscopic studies [30,33]. The parameters characterizing the asteroseismic model are shown in column 3 .

In table 2 we compare the observed and theoretical periods and rates of change of the periods. As can be seen, the model reproduces very well the observed periods. This is particularly true for the case of the $113.8 \mathrm{~s}$ and $192.6 \mathrm{~s}$ periods. Note that the observed rate of change of the $113.8 \mathrm{~s}$ and $192.6 \mathrm{~s}$ periods is more than 2 and 1.25 times larger, respectively, 


\begin{tabular}{|lcc|}
\hline Quantity & Spectroscopy & Asteroseismology \\
\hline$T_{\text {eff }}[\mathrm{K}]$ & $12100 \pm 200$ & $11980 \pm 300$ \\
$M_{\star} / M_{\odot}$ & $0.727 \pm 0.062$ & $0.705 \pm 0.023$ \\
$\log g$ & $8.21 \pm 0.10$ & $8.177 \pm 0.088$ \\
$\log \left(R_{*} / R_{\odot}\right)$ & - & $-1.945 \pm 0.037$ \\
$\log \left(L_{*} / L_{\odot}\right)$ & - & $-2.622 \pm 0.046$ \\
$M_{\mathrm{He}} / M_{\star}$ & - & $7.63 \times 10^{-3}$ \\
$M_{\mathrm{H}} / M_{\star}$ & - & $3.14 \times 10^{-5}$ \\
$X_{\mathrm{C}}, X_{\mathrm{O}}$ (center) & - & $0.326,0.661$ \\
\hline
\end{tabular}

Table 1. Characteristics of L19-2 obtained using spectroscopy [30, 33] and according to the asteroseismic model [48]. The quoted uncertainties in the asteroseismic model are the internal errors of the period-fit procedure.

\begin{tabular}{|cccccc|}
\hline $\begin{array}{c}\Pi^{\mathrm{o}} \\
{[\mathrm{s}]}\end{array}$ & $\begin{array}{c}\Pi^{\mathrm{t}} \\
{[\mathrm{s}]}\end{array}$ & $\begin{array}{c}\dot{\Pi}^{\mathrm{o}} \\
{\left[10^{-15} \mathrm{~s} / \mathrm{s}\right]}\end{array}$ & $\begin{array}{c}\dot{\Pi}^{\mathrm{t}} \\
{\left[10^{-15} \mathrm{~s} / \mathrm{s}\right]}\end{array}$ & $k$ & $\ell$ \\
\hline 118.7 & $117.21(\mathrm{~T})$ & - & 0.47 & 1 & 1 \\
192.6 & 192.88 & $3.0 \pm 0.6$ & 2.41 & 2 & 1 \\
113.8 & 113.41 & $3.0 \pm 0.6$ & 1.42 & 2 & 2 \\
143.6 & 145.41 & - & 0.89 & 3 & 2 \\
\hline
\end{tabular}

Table 2. The observed and theoretical asteroseismic model periods and rates of period change. The capital letter $\mathrm{T}$ in parenthesis for the mode with $k=1, \ell=1$ stands for a mode trapped in the $\mathrm{H}$ envelope.

than the theoretically expected values. If we assume that the rate of period change of these two modes reflects the evolutionary timescale of the star, then the disagreement between the observed and theoretical values of $\dot{\Pi}$ would be a hint that L19-2 could be cooling faster than predicted by the standard theory of white dwarf evolution.

The internal chemical stratification and the propagation diagram - the run of the critical frequencies, namely the Brunt-Väisälä frequency, $N$, and the Lamb frequency, $L_{\ell}$ for $\ell=1,2$ - of our asteroseismic model are shown in figure 1. Each chemical transition region produces clear and distinctive features in $N$, which are eventually responsible for the mode trapping properties of the model $[42,57]$. In the core of the star, there is a peak at $-\log (q) \approx 0.3$ (at $q \equiv 1-M_{r} / M_{\star}$ ) resulting from steep variations in the inner CO profile which are caused by the occurrence of extra mixing episodes beyond the fully convective core during central helium burning. The apparent bump in $N^{2}$ at $-\log (q) \approx 2-3$ is caused by the chemical transition of $\mathrm{He}$ to $\mathrm{C}$ and $\mathrm{O}$ resulting from nuclear processing in the previous AGB and thermally-pulsing AGB evolutionary phases. Finally, there is the transition region between $\mathrm{H}$ and $\mathrm{He}$ at $-\log (q) \approx 4.5$, which is smoothly shaped by the action of timedependent element diffusion.

\subsection{Mode trapping}

Table 2 also reveals that the rates of period change for the various modes are substantially different. This is because the modes have distinct mode trapping properties. Briefly, mode trapping is a mechanical resonance between the local oscillation wavelength of a pulsation mode with the thickness of one of the compositional layers (for instance, the $\mathrm{H}$ envelope or 


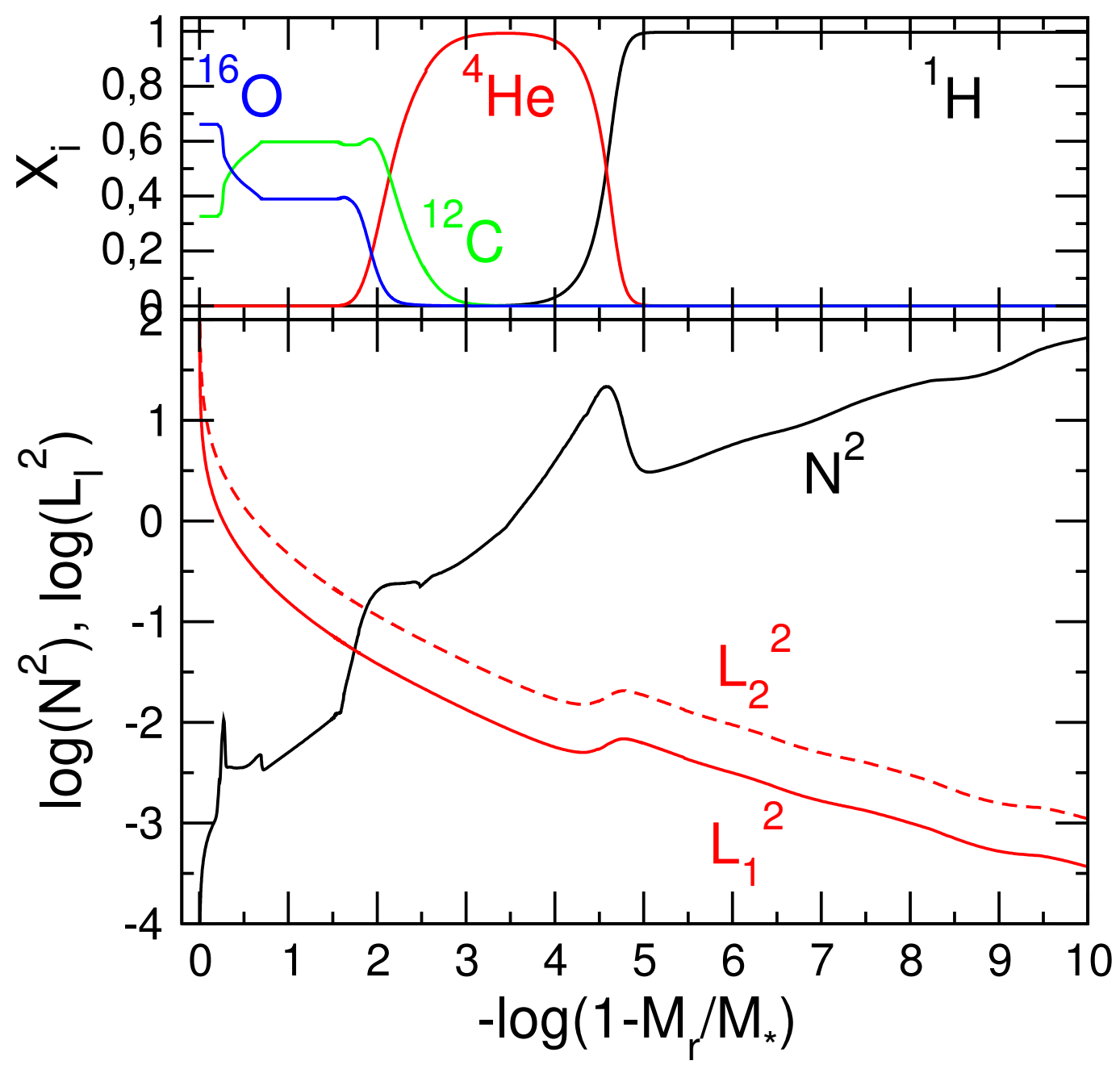

Figure 1. The internal chemical stratification (upper panel) and a propagation diagram (lower panel) of our asteroseismic model for L19-2, characterized by $M_{\star}=0.705 M_{\odot}$ and $T_{\text {eff }}=12033 \mathrm{~K}$.

the He buffer). Mode trapping can reduce the rate of period change of a mode by up to a factor of 2 if it is trapped in the outer $\mathrm{H}$ envelope [42]. The trapping properties of pulsation modes can be studied [56] by examining their radial eigenfunction $\left(y_{1}=\xi_{r} / r\right)$ and weight function, $w$. Figure 2 shows these functions for the modes with $(k, \ell)=(1,1),(2,1),(2,2)$, and $(3,2)$ of the asteroseismic model of L19-2. The weight function of a given mode allows one to infer the regions of the star that most contribute to the period formation [58]. Clearly, $y_{1}$ and $w$ for the mode with $k=1, \ell=1$ have appreciable amplitudes only in the region bounded by the $\mathrm{He} / \mathrm{H}$ interface and the stellar surface, and so it is a mode strongly trapped in the outer $\mathrm{H}$ envelope. This property for the $k=1, \ell=1$ mode holds also for all the models with structural parameters $\left(M_{\star}, M_{\mathrm{H}}, T_{\text {eff }}\right)$ similar to those of the best-fit model. Since the region where this mode is relevant is located close to the surface, gravitational contraction (that is still appreciable in these regions) acts reducing the period change due to cooling alone [42]. This is the reason why the rate of period change of the $k=1, \ell=1$ ( $\Pi \sim 118 \mathrm{~s})$ mode is small. In contrast, the non-trapped modes with $k=2, \ell=2(\Pi \sim 113 \mathrm{~s})$ and $k=2, \ell=1(\Pi \sim 192 \mathrm{~s})$ have amplitudes below the $\mathrm{He} / \mathrm{H}$ interface and, in particular, at 
the $\mathrm{He} / \mathrm{C} / \mathrm{O}$ chemical transition region. Finally, the mode with $k=3, \ell=2(\Pi \sim 143 \mathrm{~s})$ has mode-trapping properties which are intermediate between those of the trapped and nontrapped modes. Since non-trapped modes are not affected by gravitational contraction, they have larger rates of period change.

In summary, the rates of period change for the $k=2, \ell=2(\Pi \sim 113 \mathrm{~s})$ and $k=$ $2, \ell=1(\Pi \sim 192 \mathrm{~s})$ modes are not affected by gravitational contraction, implying that they are sensitive to cooling and are useful for our analysis. In addition, given the observed characteristics of the L19-2 pulsation spectrum, these two modes are the only suitable ones for investigating long term period changes.

We point that, despite the fact that the asteroseismic best-fit model reproduces the observed periods quite well (see table 2), the predicted $\dot{\Pi}$ values for the $192.6 \mathrm{~s}$ and 113.8 $\mathrm{s}$ modes differ by a factor $(2.41 / 1.42) \sim 1.7$. On the other hand, taking into account the observational $\dot{\Pi}$ values and error bars, this factor is actually $\frac{(3.0+0.6)}{(3.0-0.6)}=1.5$. In other words, our asteroseismic model does not match the observed rate of period change of both modes simultaneously. In the next section, we discuss some probable sources of uncertainty affecting the theoretical rates of period change.

\subsection{Uncertainties in the theoretical value of $\dot{\Pi}$}

Several sources of uncertainty affect the value of the rates of period change for the $k=2, \ell=1$ and $k=2, \ell=2$ modes of our asteroseismic model. This is a crucial point to estimate the uncertainties in the derived axion mass. We consider two main sources of errors. The first one is the poorly known ${ }^{12} \mathrm{C}(\alpha, \gamma){ }^{16} \mathrm{O}$ nuclear reaction rate at relevant stellar energies, whereas the second one stems from the uncertainties in the parameters of the asteroseismic model $\left(M_{\star}, T_{\text {eff }}\right.$, and $\left.M_{\mathrm{H}}\right)$.

The ${ }^{12} \mathrm{C}(\alpha, \gamma){ }^{16} \mathrm{O}$ nuclear reaction plays a crucial role in shaping the chemical structure of the cores of white dwarfs. Indeed, the final carbon-oxygen stratification of a newly born white dwarf strongly depends on the efficiency of this reaction rate during the late stages of core helium burning [59]. In our evolutionary calculations, we employed the most commonly used rate for the ${ }^{12} \mathrm{C}(\alpha, \gamma){ }^{16} \mathrm{O}$ rate [60]. Unfortunately, the rate of this reaction is not accurately known. Estimated uncertainties of this reaction rate have been compiled [61]. The uncertainties vary from a factor $\sim 1.4$ to a factor $\sim 0.6$. The uncertainties of the theoretical rates of period change of the asteroseismic model of the ZZ Ceti star G117-B15A due to the uncertainties in the ${ }^{12} \mathrm{C}(\alpha, \gamma){ }^{16} \mathrm{O}$ reaction rate have also been estimated [39]. Not surprisingly, the value of $\dot{\Pi}$ for a mode trapped in the $\mathrm{H}$ envelope exhibits a modest variation, of about $2.5 \%$, when the central ${ }^{16} \mathrm{O}$ abundance strongly increases from $X_{16} \mathrm{O}=0.482$ to $X_{16} \mathrm{O}=0.795$. At variance with this, in the case of non-trapped modes, the rate of period change experiences quite large changes, of up to $\sim 60 \%$. These results allow us to assess in an approximate way the impact of the uncertainties of the ${ }^{12} \mathrm{C}(\alpha, \gamma){ }^{16} \mathrm{O}$ reaction rate on the value of the rate of period change of the non-trapped modes of interest in L19-2, that is, the $k=2, \ell=1$ and $k=2, \ell=2$ modes. We adopt an uncertainty $\varepsilon_{1} \sim 0.85 \times 10^{-15} \mathrm{~s} / \mathrm{s}$ $\left(\varepsilon_{1} \sim 1.45 \times 10^{-15} \mathrm{~s} / \mathrm{s}\right)$ for the $\dot{\Pi}^{\mathrm{t}}$ associated with the period $\sim 113 \mathrm{~s}(\sim 192 \mathrm{~s})$.

Another source of error in the theoretical values of $\dot{\Pi}$ comes from the uncertainties in the asteroseismic model for L19-2. These are internal errors of the period-fit procedure [39]. We estimate that the uncertainty in the rate of period change for the modes with $k=2, \ell=1$ and $k=2, \ell=2$ is $\varepsilon_{2} \sim 0.06 \times 10^{-15} \mathrm{~s} / \mathrm{s}$ at most [39]. This is a small uncertainty that is completely negligible when compared to the uncertainties arising from the poorly known ${ }^{12} \mathrm{C}(\alpha, \gamma){ }^{16} \mathrm{O}$ reaction rate. 

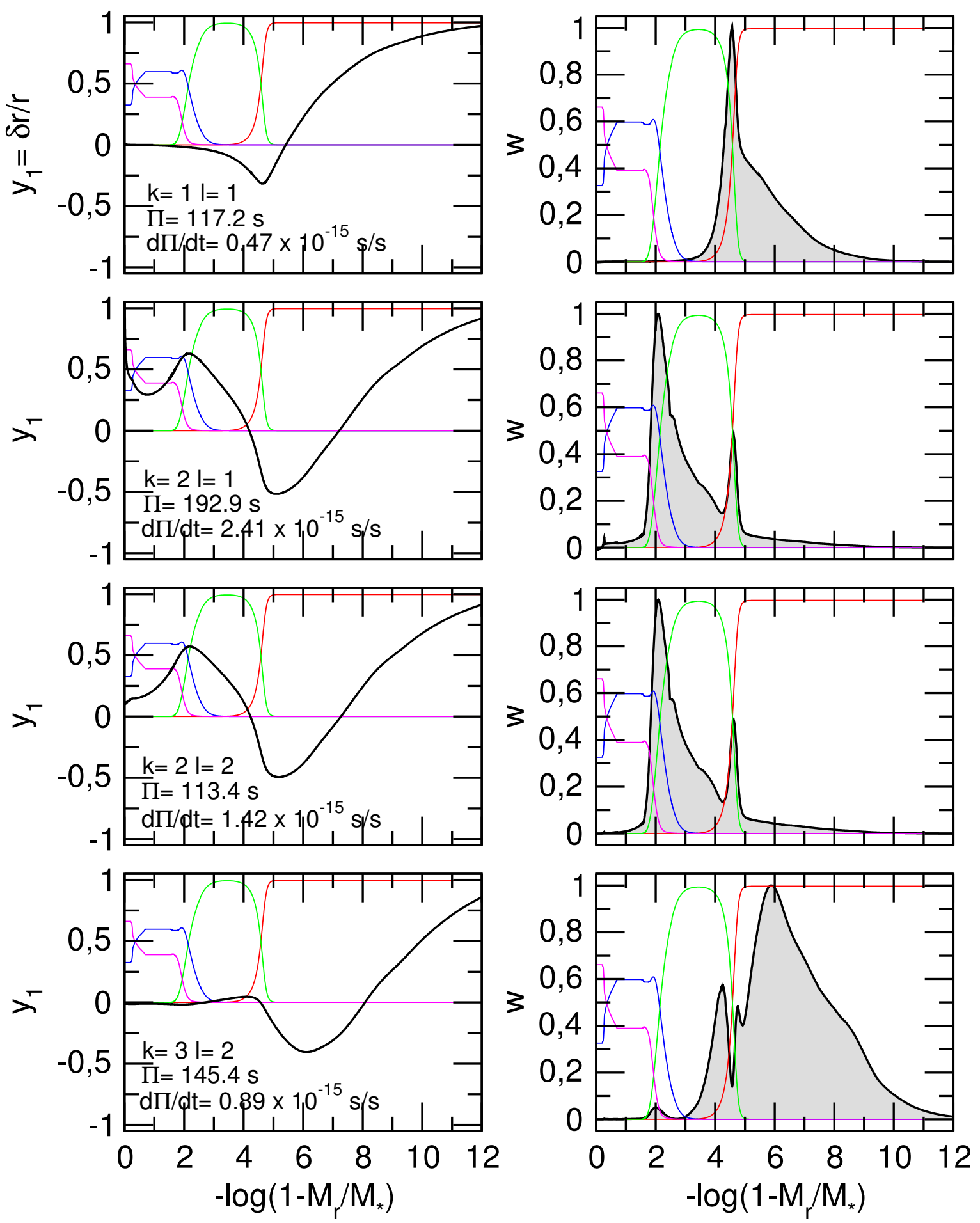

Figure 2. The radial eigenfunction $y_{1}=\xi_{r} / r$ (left panels) and the normalized weight function $w$ (right panels, shaded) in terms of the outer mass fraction, corresponding to the modes with $(k, \ell)=(1,1),(2,1),(2,2)$, and $(3,2)$ (from top to bottom) of the asteroseismic model. The chemical abundances are also shown, to provide a reference. 
In addition to the ${ }^{12} \mathrm{C}(\alpha, \gamma){ }^{16} \mathrm{O}$ reaction rate and the period-fit procedure, other possible sources of uncertainty are the amount of mixing that occurs during both the core $\mathrm{He}$ burning and thermally pulsing AGB phases, the metallicity of the white dwarf progenitor, rotation, etc. We caution that these sources of uncertainty (which have been neglected in our treatment) could affect to some extent the values of the theoretical rates of period change. The precise assessment of the impact of these uncertainties on $\dot{\Pi}$ is beyond the scope of the present paper but will be addressed in a future publication.

\section{Impact of axion emission on the rates of period change}

In the previous section we have presented results of periods and rates of period change for L19-2 that do not take into account energy sources other than gravothermal energy for the evolutionary cooling of the star. We have found that the theoretically expected rates of period change of the modes with $k=2, \ell=1$ and $k=2, \ell=2$ are smaller than the $\dot{\Pi}$ value measured for the periods at $192 \mathrm{~s}$ and $113 \mathrm{~s}$ of L19-2, suggesting the existence of some additional cooling mechanism in this star. Hereafter, we shall assume that this additional cooling can be entirely attributed to the emission of axions.

We computed a set of DA white dwarf cooling sequences incorporating axion emission $[35,39,41]$. This was done considering different axion masses and the same structural parameters $\left(M_{\star}, M_{\mathrm{H}}\right)$ as those of the asteroseismic model discussed above. We adopted a range of values for the mass of the axion $0 \leq m_{\mathrm{a}} \cos ^{2} \beta \leq 30 \mathrm{meV}$ with a step size $\Delta m_{\mathrm{a}}=1 \mathrm{meV}$. In all the cases we employed the most reliable axion emission rates [18]. The evolutionary calculations including the emission of axions were started at evolutionary stages long before the ZZ Ceti phase to ensure that the cumulative effects of axion emission reached an equilibrium value before the theoretical models have the effective temperature of L19-2.

We computed the pulsation periods and the rates of period change of the asteroseismic model $\left(T_{\text {eff }} \sim 12030 \mathrm{~K}\right)$ for increasing values of $m_{\mathrm{a}}$. The variation in the periods is negligible, in spite of the rather wide range of axion masses considered. This is an important property as previously noted [35], that allows one to put constraints on the mass of the axion. The rates of period change for the modes with $k=1,2, \ell=1$, and $k=2,3, \ell=2$ of the asteroseismic model for increasing values of $m_{\mathrm{a}}$ are displayed in figure 3 . At odds with what happens with the pulsation periods, the values of $\dot{\Pi}^{t}$ are strongly affected by the additional cooling source, substantially increasing for increasing values of $m_{\mathrm{a}}$. In particular, the rate of period change of the modes with $k=2, \ell=1$ and $k=2, \ell=2$, which are the relevant ones in the present analysis, increase by a factor of about 4 for the range of axion masses considered here.

\section{The axion mass}

Here, we focus on the modes with $k=2, \ell=1$ and $k=2, \ell=2$, for which we have a measurement of their rate of period change. In figure 4 we show the theoretical value of $\dot{\Pi}$ corresponding to the period $\Pi \sim 113 \mathrm{~s}$ for increasing values of the axion mass (blue solid curve). The dashed curves embracing the solid curve represent the estimated uncertainty in the theoretical value of $\dot{\Pi}, \varepsilon_{\dot{\Pi}}=0.85 \times 10^{-15} \mathrm{~s} / \mathrm{s}$. This value was obtained considering the uncertainty introduced by our lack of precise knowledge of the ${ }^{12} \mathrm{C}(\alpha, \gamma){ }^{16} \mathrm{O}$ reaction rate $\left(\varepsilon_{1} \sim 0.85 \times 10^{-15} \mathrm{~s} / \mathrm{s}\right)$, which completely dominates the errors in the asteroseismic model $\left(\varepsilon_{2} \sim 0.06 \times 10^{-15} \mathrm{~s} / \mathrm{s}\right.$, see section 3.2). We are assuming that the uncertainty for the case in which $m_{\mathrm{a}}>0$ is the same as that computed for the case in which $m_{\mathrm{a}}=0$. If 


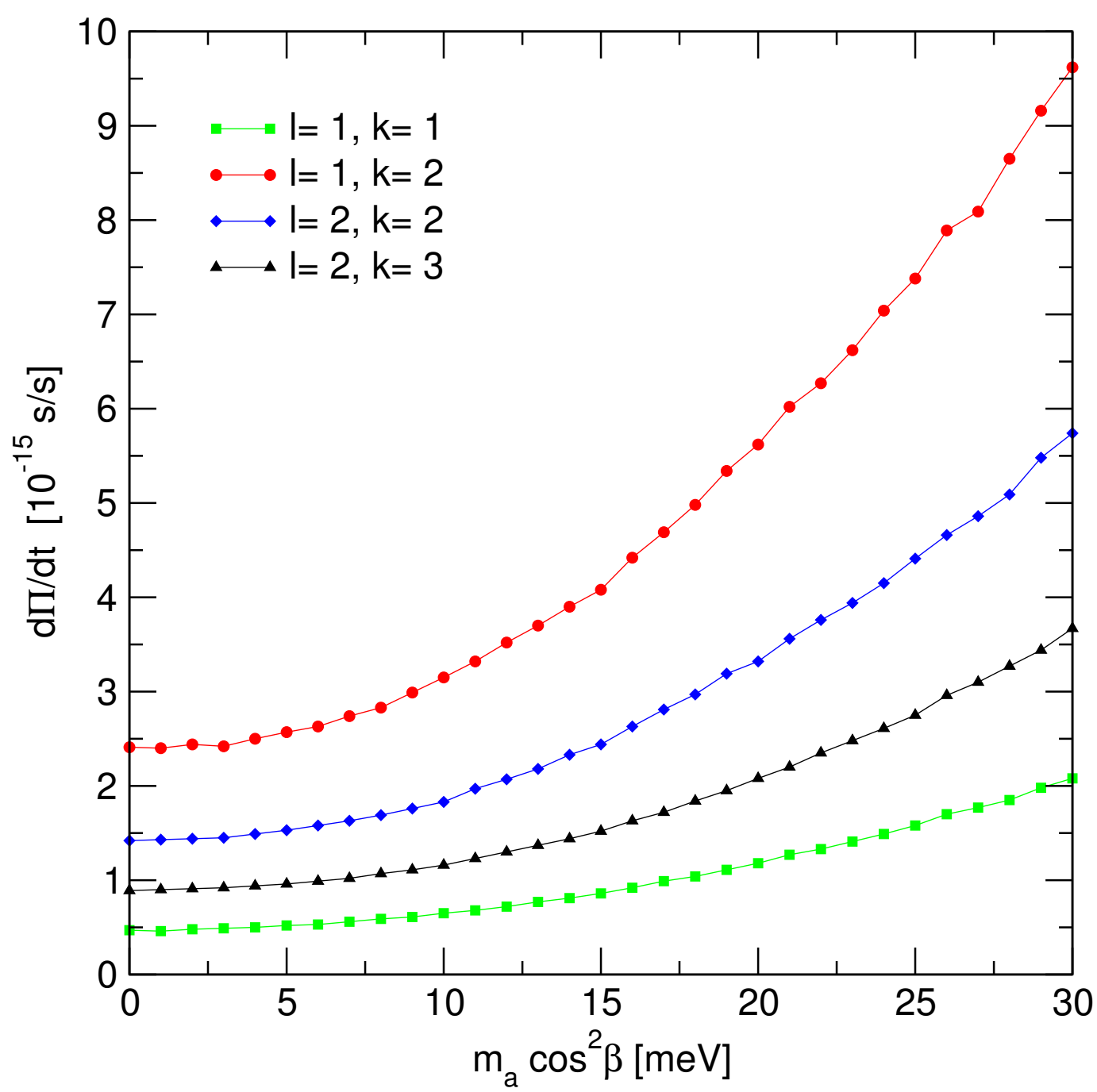

Figure 3. The rates of period change of the modes with $k=1,2, \ell=1$ and $k=2,3, \ell=2$, corresponding to our asteroseismic model for L19-2 in terms of the axion mass.

we consider one standard deviation from the observational value, we obtain an upper limit on the axion mass of $m_{\mathrm{a}} \cos ^{2} \beta \lesssim 25 \mathrm{meV}\left(g_{\mathrm{ae}}=7 \times 10^{-13}\right)$. If, instead, we consider the mode with period $\Pi \sim 192 \mathrm{~s}$, we obtain the results displayed in figure 5 . In this case, the dashed curves embracing the solid red curve represent the uncertainty in the theoretical value of $\dot{\Pi}, \varepsilon_{\dot{\Pi}}=1.45 \times 10^{-15} \mathrm{~s} / \mathrm{s}$ (section 3.2). We obtain an upper limit for the axion mass of $m_{\mathrm{a}} \cos ^{2} \beta \lesssim 18 \mathrm{meV}\left(g_{\mathrm{ae}}=5.1 \times 10^{-13}\right)$.

In summary, based on the rates of period change measured for the ZZ Ceti star L19-2, we derive an upper limit of $m_{\mathrm{a}} \cos ^{2} \beta$ of $25.1 \mathrm{meV}\left(g_{\mathrm{ae}}=7.0 \times 10^{-13}\right)$. We note that, if the uncertainties of the theoretical rates of period change were drastically reduced, then the upper limit on the axion mass would be lowered $(\sim 13-21 \mathrm{meV})$.

\section{Discussion and conclusions}

In this paper we have derived new bounds for the mass of the hypothetical particle called axion. For this purpose we used a detailed asteroseismic model for L19-2, a hot and relatively 


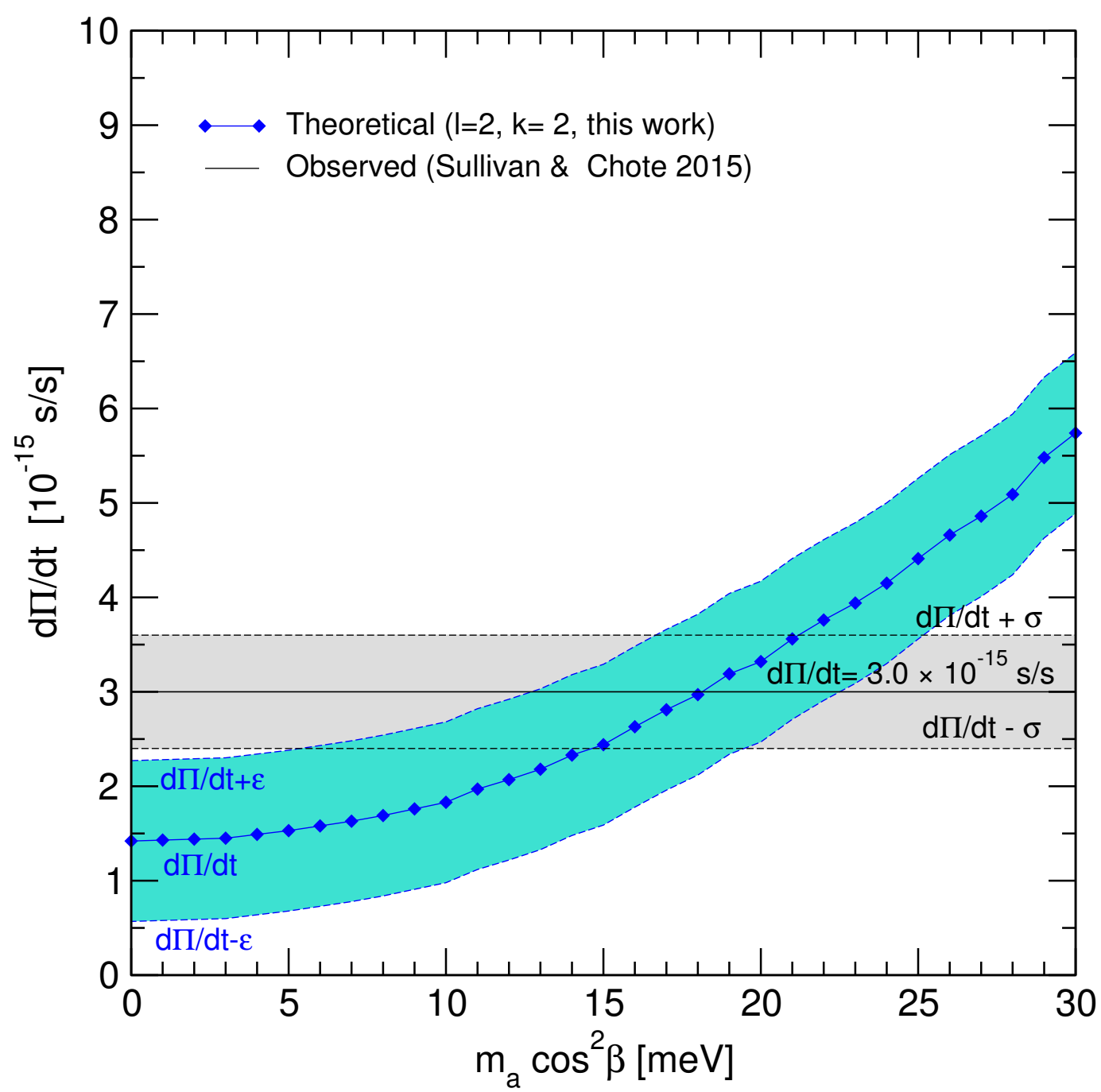

Figure 4. The rate of period change for the mode with $k=2, \ell=2(\Pi \sim 113 \mathrm{~s})$ of our asteroseismic model in terms of the axion mass (solid blue curve with diamonds). Dashed blue curves represent the errors in $\dot{\Pi}$ due to internal uncertainties in the modeling and in the asteroseismic procedure $\left(\varepsilon \sim 0.85 \times 10^{-15} \mathrm{~s} / \mathrm{s}\right)$. The horizontal solid black line indicates the observed value and the dashed black lines its corresponding uncertainties [43].

massive ZZ Ceti star, derived from fully evolutionary calculations of DA white dwarfs [48], and we employed the recent determination of the rate of period change for the largest-amplitude modes $(k=2, \ell=2, \Pi \approx 113 \mathrm{~s}$ and $k=2, \ell=1, \Pi \approx 192 \mathrm{~s})$ of this star [43].

We first compared the observed rate of period change for these modes, $\dot{\Pi}=(3.0 \pm 0.6) \times$ $10^{-15} \mathrm{~s} / \mathrm{s}$, with the rates of period change of our asteroseismic model, $\dot{\Pi}^{\mathrm{t}}=(1.42 \pm 0.85) \times$ $10^{-15} \mathrm{~s} / \mathrm{s}(k=2, \ell=2)$ and $\dot{\Pi}^{\mathrm{t}}=(2.41 \pm 1.45) \times 10^{-15} \mathrm{~s} / \mathrm{s}(k=2, \ell=1)$, computed under the assumption that the cooling of this star is governed only by the release of gravothermal energy. The fact that the observed value is about 1.25-2 times larger than the theoretically expected ones hints at the possibility that this star might be cooling faster than the standard theory of white dwarf evolution predicts. We took into account the possible sources of uncertainties in the theoretical values of the rate of period change. The uncertainties affecting 


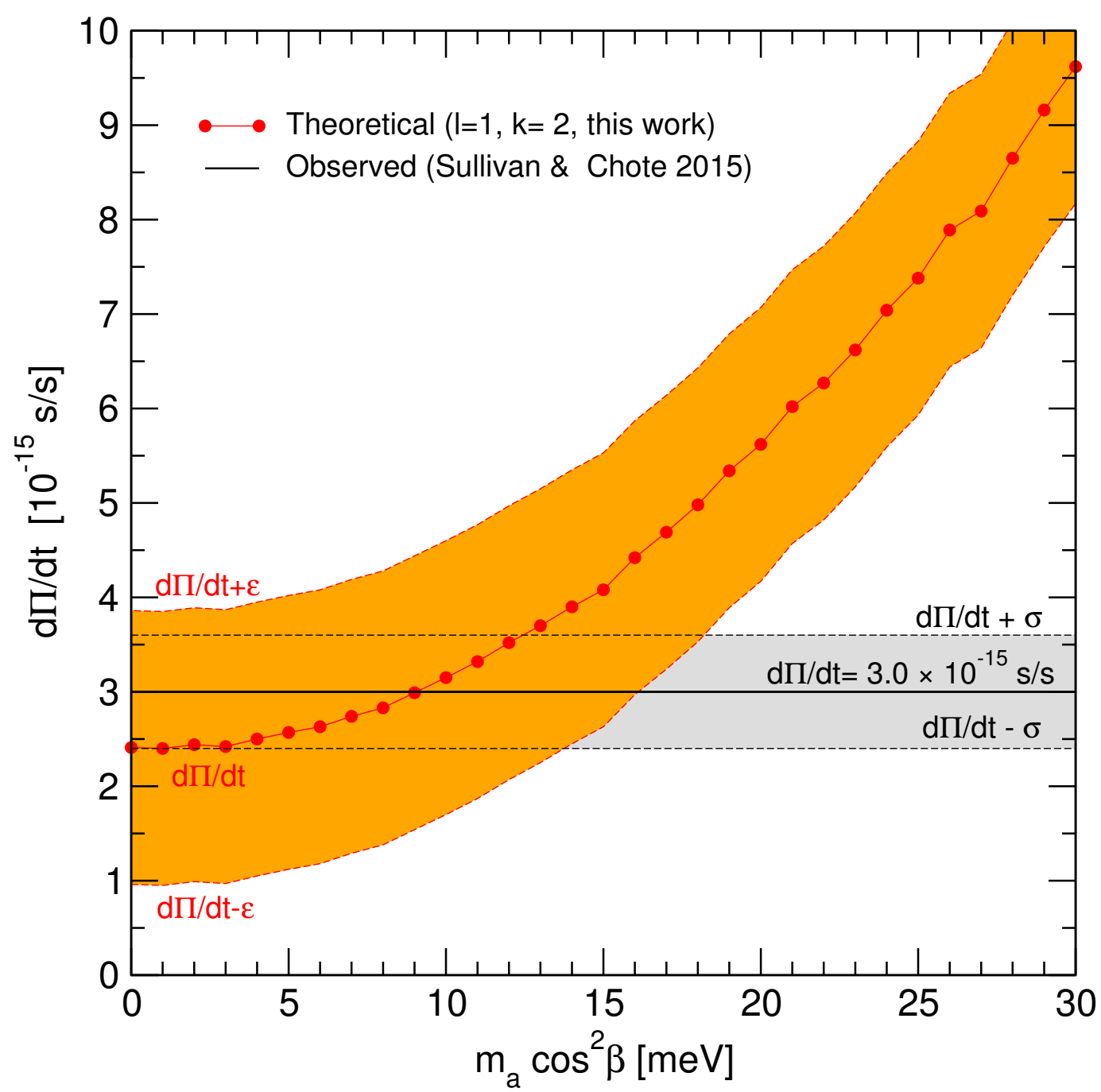

Figure 5. Similar to figure 4 , but for the mode with $k=2, \ell=1(\Pi \sim 192 \mathrm{~s})$. In this case, the theoretical uncertainty is of the rate of period change is $\varepsilon \sim 1.45 \times 10^{-15} \mathrm{~s} / \mathrm{s}$.

the poorly determined ${ }^{12} \mathrm{C}(\alpha, \gamma){ }^{16} \mathrm{O}$ reaction rate have a strong impact on the theoretical value of the rates of period change for the two modes of interest, because these modes have non-negligible amplitudes at the core regions of the asteroseismic model. These uncertainties largely overcome the internal uncertainties of the asteroseismic model. We reached the conclusion that the uncertainties in the theoretical rates of period change are of $\sim 60 \%$.

Next, we assumed that the additional cooling necessary to account for the large observed rate of period change of L19-2 could be attributed to axion emission. Then, we introduced axion emission in our asteroseismic model, considering a sufficiently large range of axion masses (between 0 and $30 \mathrm{meV}$ ). We found that the periods do not change, but the rates of period change are strongly affected by the precise value of the axion mass. We found that the mass of axion necessary to account for the observed rate of period change could be as high as $\sim 18 \mathrm{meV}$ or $\sim 25 \mathrm{meV}$, depending on the mode we use in our analysis. Specifically, when the mode with period $\sim 113 \mathrm{~s}$ was employed, we obtained $5 \lesssim m_{\mathrm{a}} \cos ^{2} \beta \lesssim 25$, or, in terms of the axion-electron coupling constant, $1.4<g_{\text {ae }} / 10^{-13}<7$ (figure 4 ). In the case 


\begin{tabular}{|cccc|}
\hline Method & Object (class) & $m_{\mathrm{a}} \cos ^{2} \beta[\mathrm{meV}]$ & Reference \\
\hline Asteroseismology & G117-B15A (DAV) & $\lesssim 10$ & {$[28]$} \\
Asteroseismology & G117-B15A (DAV) & $\lesssim 4.4$ & {$[35]$} \\
Asteroseismology & G117-B15A (DAV) & $\lesssim 26.5$ & {$[36]$} \\
Asteroseismology & G117-B15A (T, DAV) & $14.7-19.7$ & {$[39]$} \\
Asteroseismology & R548 (T, DAV) & $11.3-21.4$ & {$[41]$} \\
Asteroseismology & PG 1351+489 (NT, DBV) & $\lesssim 19.5$ & {$[62]$} \\
Asteroseismology & L19-2 (NT, DAV) & $\lesssim 25$ & This work \\
\hline WDLF & Observed WDLF & $\lesssim 5$ & {$[20]$} \\
WDLF & Observed WDLF & $\lesssim 6$ & {$[21]$} \\
WDLF & Observed WDLF & $\lesssim 10$ & {$[22]$} \\
\hline
\end{tabular}

Table 3. Summary of the different constraints on the DFSZ axion mass from white dwarf stars.

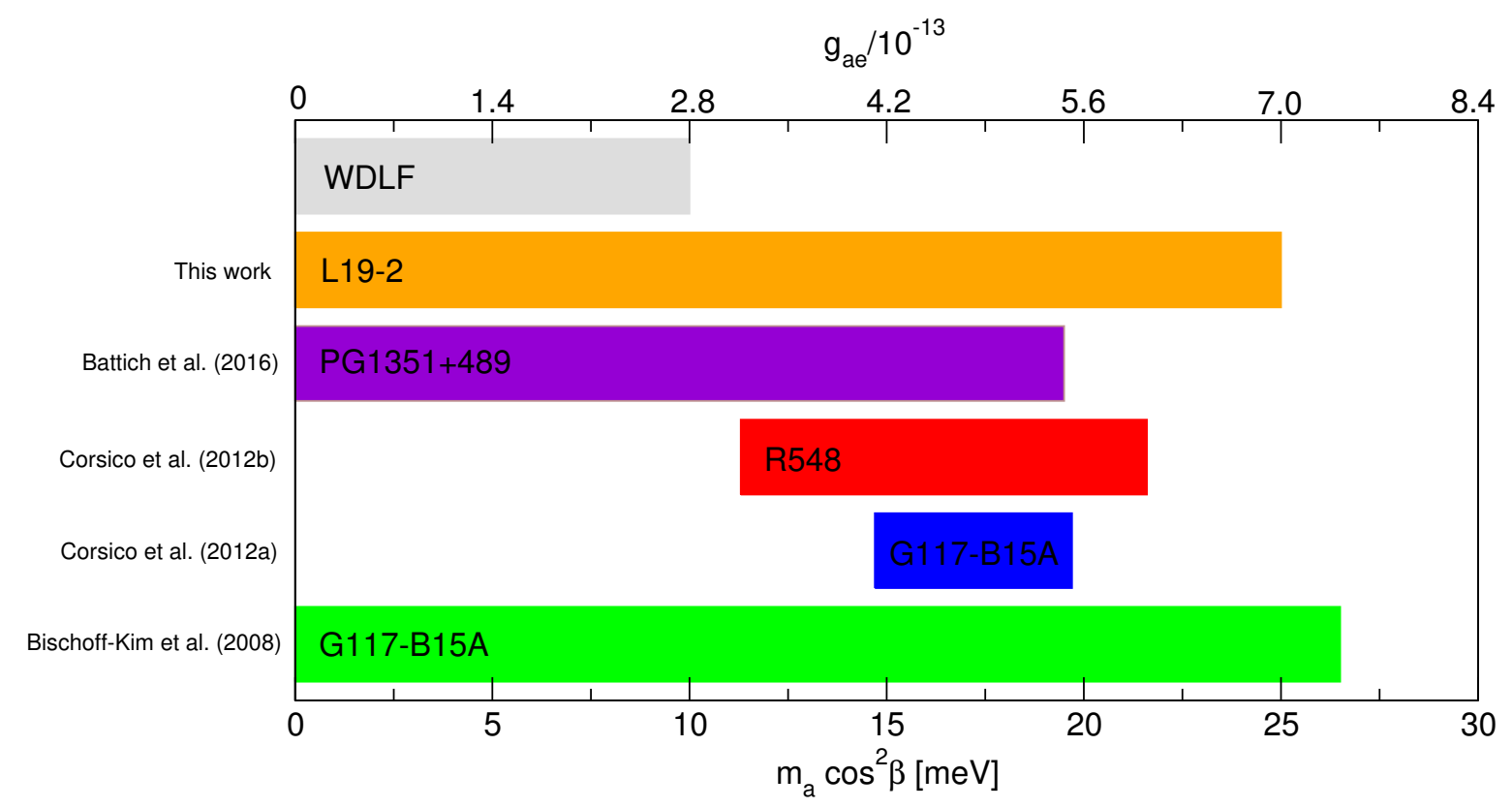

Figure 6. The recent asteroseismic inferences of the axion mass [36, 39, 41, 62] and the derivations of $m_{\mathrm{a}}$ employing the white dwarf luminosity function [20-22] (see table 3).

of the mode with period at $\sim 192 \mathrm{~s}$ we derived an upper bound for $m_{\mathrm{a}} \cos ^{2} \beta$ of $\sim 18 \mathrm{meV}$ $\left(g_{\mathrm{ae}} \sim 5 \times 10^{-13}\right)$, although a zero mass for the axion cannot be discarded (see figure 5$)$.

In table 3 we summarize the various limits on the DFSZ axion mass derived from white dwarfs using pulsating stars (top section) as well as employing the white dwarf luminosity function (bottom section), and including the results derived in the present paper. The different axion-mass limits are illustrated in figure 6 . It is worth noting that the measured value of the rate of period change of the 215 s mode of G117-B15A has been substantially changing over the years due to the introduction of corrections, like the proper motion correction, and the fact that the phase of the periodicity is affected by jumps caused by low-amplitude modes, contamination by G117-B15B (the companion star), use of different telescopes and apertures, 
and several other subtle effects [63], until it reached a value of $\dot{\Pi}=(4.19 \pm 0.73) \times 10^{-15} \mathrm{~s} / \mathrm{s}$ according to the last measurement [38]. In table 3, a label "T" ("NT") in parenthesis means that the mode for which the rate of period change has been measured is (is not) a mode trapped in the outer envelope of the star. In particular, the mode at $\sim 215 \mathrm{~s}(k=2, \ell=1)$ of G117-B15A and the mode at $\sim 212 \mathrm{~s}(k=2, \ell=1)$ of R548 are trapped modes. We remind here that the bounds on the axion mass derived using these trapped modes [39, 41] crucially depend on the nature of the mode. Specifically, a trapped mode has a theoretical rate of period change that is smaller than the values corresponding to the adjacent, non trapped modes. A small value of the theoretical $\dot{\Pi}$ results in a large axion mass [39]. It has been argued [22] that this is the origin of the discrepancy between the value of $m_{\mathrm{a}}$ derived from the pulsating stars G117-B15A and R548 and the limits inferred from the white dwarf luminosity function [20-22], that discard axion masses larger than $\sim 10 \mathrm{meV}$. Interestingly, for the case of the pulsating white dwarf L19-2 analyzed in the present paper, the modes with periods $\sim 113 \mathrm{~s}$ and $\sim 192 \mathrm{~s}$, for which a rate of period change of $\dot{\Pi}=(3.0 \pm 0.6) \times 10^{-15} \mathrm{~s} / \mathrm{s}$ has been measured [43], seem to be non-trapped modes, according to our asteroseismic model. However, our analysis yet supports upper limits for the axion mass that are substantially higher than the largest bounds obtained by using the white dwarf luminosity function $(\sim 10 \mathrm{meV})$. Note that, even if the uncertainties in the theoretical rates of period change were reduced to zero, we would obtain upper limits for the mass of the axion that are still above the maximum mass allowed by studies based on the luminosity function of white dwarfs. Nevertheless, it should be kept in mind that the errors in the observational determination of the white dwarf luminosity function are poissonian. When the remaining uncertainties in the determination of this function are taken into account this upper bound considerably weakens.

In the same vein, attention has been drawn [63] to the possibility of obtaining bounds to the axion mass from pulsating white dwarfs with He-rich atmospheres, the so-called DBV class of variable white dwarfs. Recently, an upper limit to the mass of the axion of $m_{\mathrm{a}} \cos ^{2} \beta \sim$ $19.5 \mathrm{meV}\left(g_{\mathrm{ae}} \sim 5.5 \times 10^{-13}\right)$ has been obtained [62] using the DBV star PG 1351+489 for which a value of $\dot{\Pi}=(2.0 \pm 0.9) \times 10^{-13} \mathrm{~s} / \mathrm{s}$ has been derived [64]. This upper limit seems to be in line with the predictions of the analysis based on the luminosity function of white dwarfs, with the previous ones derived employing G117-B15A and R548 [36, 39, 41], and also with those obtained in this paper for L19-2.

In view of the above discussion, and taking into account the uncertainties still plaguing both methods, it seems that the results obtained from the luminosity function of white dwarfs and asteroseismic models of variable of white dwarfs are concordant at the $1 \sigma$ level, although additional efforts are necessary to reduce the discrepancies. More measurements of the rates of period change for other pulsating white dwarfs, together with improvements in the asteroseismic models to reduce the uncertainties coming from the previous evolution, will enable the determination of new and more precise bounds on the axion mass. At the same time, additional work will be necessary as well to reduce the discrepancies between the different observed white dwarf luminosity functions, along with better estimates of the statistical uncertainties, and more reliable cooling sequences [22]. All these future improvements, will allow us to better constrain the axion mass.

\section{Acknowledgments}

We wish to thank our anonymous referee for the constructive comments and suggestions that improved the original version of the paper. Part of this work was supported by AGENCIA 
through the Programa de Modernización Tecnológica BID 1728/OC-AR, by the PIP 112200801-00940 grant from CONICET, by MINECO grant AYA2014-59084-P, by the AGAUR, by CNPq and PRONEX-FAPERGS/CNPq (Brazil). This research has made use of NASA Astrophysics Data System.

\section{References}

[1] A. Bohr and B. Mottelson, Moments of inertia of rotating nuclei, Kong. Dan. Vid. Sel. Mat. Fys. Med. 30 (1955) 1 [INSPIRE].

[2] W. Pauli, L. Rosenfeld and V. Weisskopf, Niels Bohr and the Development of Physics, Pergamon Press, New York U.S.A. (1955).

[3] M. Kobayashi and T. Maskawa, CP Violation in the Renormalizable Theory of Weak Interaction, Prog. Theor. Phys. 49 (1973) 652 [InSPIRE].

[4] J.E. Kim and G. Carosi, Axions and the Strong CP Problem, Rev. Mod. Phys. 82 (2010) 557 [arXiv:0807.3125] [INSPIRE].

[5] G.G. Raffelt, Stars as laboratories for fundamental physics: the astrophysics of neutrinos, axions, and other weakly interacting particles, University of Chicago Press, Chicago U.S.A. (1996).

[6] R.D. Peccei and H.R. Quinn, CP Conservation in the Presence of Instantons, Phys. Rev. Lett. 38 (1977) 1440 [INSPIRE].

[7] S. Weinberg, A New Light Boson?, Phys. Rev. Lett. 40 (1978) 223 [inSPIRE].

[8] F. Wilczek, Problem of Strong $p$ and $t$ Invariance in the Presence of Instantons, Phys. Rev. Lett. 40 (1978) 279 [INSPIRE].

[9] G.G. Raffelt, Axions: Motivation, limits and searches, J. Phys. A 40 (2007) 6607 [hep-ph/0611118] [INSPIRE].

[10] J.E. Kim, Weak Interaction Singlet and Strong CP Invariance, Phys. Rev. Lett. 43 (1979) 103 [INSPIRE].

[11] M.A. Shifman, A.I. Vainshtein and V.I. Zakharov, Can Confinement Ensure Natural CP Invariance of Strong Interactions?, Nucl. Phys. B 166 (1980) 493 [InSPIRE].

[12] M. Dine, W. Fischler and M. Srednicki, A Simple Solution to the Strong CP Problem with a Harmless Axion, Phys. Lett. B 104 (1981) 199 [inSPIRE].

[13] A.R. Zhitnitsky, On Possible Suppression of the Axion Hadron Interactions. (In Russian), Sov. J. Nucl. Phys. 31 (1980) 260 [Yad. Fiz. 31 (1980) 497] [inSPIRE].

[14] J. Redondo, Axions at the International Axion Observatory, arXiv:1601.00578 [INSPIRE].

[15] L.G. Althaus, A.H. Córsico, J. Isern and E.G. a Berro, Evolutionary and pulsational properties of white dwarf stars, Astron. Astrophys. Rev. 18 (2010) 471 [arXiv:1007.2659] [InSPIRE].

[16] G.G. Raffelt, Axion Constraints From White Dwarf Cooling Times, Phys. Lett. B 166 (1986) 402 [INSPIRE].

[17] L. Mestel, On the theory of white dwarf stars. I. The energy sources of white dwarfs, Mon. Not. Roy. Astron. Soc. 112 (1952) 583.

[18] M. Nakagawa, Y. Kohyama and N. Itoh, Axion Bremsstrahlung in Dense Stars, Astrophys. J. 322 (1987) 291 [INSPIRE].

[19] M. Nakagawa, T. Adachi, Y. Kohyama and N. Itoh, Axion bremsstrahlung in dense stars. II Phonon contributions, Astrophys. J. 326 (1988) 241. 
[20] J. Isern, E. García-Berro, S. Torres and S. Catalán, Axions and the cooling of white dwarf stars, Astrophys. J. 682 (2008) L109 [arXiv:0806.2807] [InSPIRE].

[21] J. Isern, S. Catalán, E. García-Berro and S. Torres, Axions and the white dwarf luminosity function, J. Phys. Conf. Ser. 172 (2009) 012005 [arXiv:0812.3043] [INSPIRE].

[22] M.M. Miller Bertolami, B.E. Melendez, L.G. Althaus and J. Isern, Revisiting the axion bounds from the Galactic white dwarf luminosity function, JCAP 10 (2014) 069 [arXiv:1406.7712] [INSPIRE].

[23] B.G. Castanheira, S.O. Kepler, S.J. Kleinman, A. Nitta and L. Fraga, Discovery of five new massive pulsating white dwarf stars, Mon. Not. Roy. Astron. Soc. 430 (2013) 50.

[24] W. Unno, Y. Osaki, H. Ando, H. Saio and H. Shibahashi, Nonradial oscillations of stars, University of Tokyo Press, Tokyo Japan (1989).

[25] D.E. Winget and S.O. Kepler, Pulsating White Dwarf Stars and Precision Asteroseismology, Ann. Rev. Astron. Astrophys. 46 (2008) 157 [arXiv:0806.2573] [InSPIRE].

[26] G. Fontaine and P. Brassard, The Pulsating White Dwarf Stars, Publ. Astron. Soc. Pac. 120 (2008) 1043.

[27] D.E. Winget, C.J. Hansen and H.M. van Horn, Do pulsating PG1159-035 stars put constraints on stellar evolution?, Nature 303 (1983) 781.

[28] J. Isern, M. Hernanz and E. García-Berro, Axion cooling of white dwarfs, Astrophys. J. 392 (1992) L23 [INSPIRE].

[29] E.L. Robinson et al., The pulsation index, effective temperature, and thickness of the hydrogen layer in the pulsating DA white dwarf G117-B15A, Astrophys. J. 438 (1995) 908.

[30] D. Koester and N.F. Allard, The ZZ Ceti Instability Strip Revisited, Balt. Astron. 9 (2000) 119.

[31] D. Koester and J.B. Holberg, The ZZ Ceti Instability Strip, in 12th European Workshop on White Dwarfs, J.L. Provencal, H.L. Shipman, J. MacDonald and S. Goodchild eds., Ast. Soc. P. 226 (2001) 299.

[32] P. Bergeron, F. Wesemael, R. Lamontagne, G. Fontaine, R.A. Saffer and N.F. Allard, Optical and Ultraviolet Analyses of ZZ Ceti Stars and Study of the Atmospheric Convective Efficiency in DA White Dwarfs, Astrophys. J. 449 (1995) 258.

[33] P. Bergeron, G. Fontaine, M. Billeres, S. Boudreault and E.M. Green, On the purity of the ZZ Ceti instability strip: Discovery of more pulsating DA white dwarfs on the basis of optical spectroscopy, Astrophys. J. 600 (2004) 404 [astro-ph/0309483] [INSPIRE].

[34] S.O. Kepler, R.E. Nather, J.T. McGraw and E.L. Robinson, The pulsation periods of the pulsating white dwarf G117-B15A, Astrophys. J. 254 (1982) 676.

[35] A.H. Córsico, O.G. Benvenuto, L.G. Althaus, J. Isern and E. Garcia-Berro, The Potential of the variable DA white dwarf G117-B15A as a tool for fundamental physics, New Astron. 6 (2001) 197 [astro-ph/0104103] [INSPIRE].

[36] A. Bischoff-Kim, M.H. Montgomery and D.E. Winget, Strong limits on the DFSZ axion mass with G117-B15A, Astrophys. J. 675 (2008) 1512 [arXiv:0711.2041] [INSPIRE].

[37] A.D. Romero, A.H. Córsico, L.G. Althaus, S.O. Kepler, B.G. Castanheira and M.M.M. Bertolami, Toward ensemble asteroseismology of ZZ Ceti stars with fully evolutionary models, Mon. Not. Roy. Astron. Soc. 420 (2012) 1462 [arXiv:1109.6682] [INSPIRE].

[38] S.O. Kepler, White Dwarf Stars: Pulsations and Magnetism, in Progress in Solar/Stellar Physics with Helio- and Asteroseismology, H. Shibahashi, M. Takata and A.E. Lynas-Gray eds., Astron. Soc. P. 462 (2012) 322. 
[39] A.H. Córsico et al., The rate of cooling of the pulsating white dwarf star G117-B15A: a new asteroseismological inference of the axion mass, Mon. Not. Roy. Astron. Soc. 424 (2012) 2792 [arXiv:1205.6180] [INSPIRE].

[40] A.S. Mukadam et al, Measuring the Evolutionary Rate of Cooling of ZZ Ceti, Astrophys. J. 771 (2013) 17 [INSPIRE].

[41] A.H. Córsico et al., An independent limit on the axion mass from the variable white dwarf star R548, JCAP 12 (2012) 010 [arXiv:1211.3389] [INSPIRE].

[42] P.A. Bradley, Theoretical Models for Asteroseismology of DA White Dwarf Stars, Astrophys. J. 468 (1996) 350.

[43] D.J. Sullivan and P. Chote, The Frequency Stability of the Pulsating White Dwarf L19-2, in 19th European Workshop on White Dwarfs, P. Dufour et al. eds., Astron. Soc. P. 493 (2015) 199.

[44] D.J. Sullivan, The Pulsating White Dwarf L19-2, Bal. Astron. 7 (1998) 159.

[45] P.A. Bradley, Asteroseismological Constraints on the Structure of the ZZ Ceti Stars L19-2 and GD 165, Astrophys. J. 552 (2001) 326.

[46] B.G. Castanheira and S.O. Kepler, Seismological studies of ZZ Ceti stars - II. Application to the ZZ Ceti class, Mon. Not. Roy. Astron. Soc. 396 (2009) 1709.

[47] A. D. Romero, A. H. Córsico, L. G. Althaus, S. O. Kepler, B. G. Castanheira and M. M. Miller Bertolami, Toward ensemble asteroseismology of ZZ Ceti stars with fully evolutionary models, Mon. Not. Roy. Astron. Soc. 420 (2012) 1462-1480

[48] A.D. Romero, S.O. Kepler, A.H. Córsico, L.G. Althaus and L. Fraga, Asteroseismological Study of Massive ZZ Ceti Stars with Fully Evolutionary Models, Astrophys. J. 779 (2013) 58 [arXiv: 1310.4137].

[49] J.T. McGraw, Southern hemisphere ZZ Ceti stars: the new variable L19-2 and BPM 30551, Astrophys. J. Lett. 214 (1977) L123.

[50] D.E. O'Donoghue and B. Warner, Observations and analysis of the low amplitude ZZ Ceti variable, L19-2, Mon. Not. Roy. Astron. Soc. 200 (1982) 563.

[51] D. O'Donoghue and B. Warner, The period structure and stability of the pulsating white dwarf L19-2, Mon. Not. Roy. Astron. Soc. 228 (1987) 949.

[52] R.E. Nather, D.E. Winget, J.C. Clemens, C.J. Hansen and B.P. Hine, The whole earth telescope - A new astronomical instrument, Astrophys. J. 361 (1990) 309.

[53] D.J. Sullivan et al., in preparation.

[54] G. Pajdosz, Non-evolutionary secular period increase in pulsating DA white dwarfs, Astron. Astrophys. 295 (1995) L17.

[55] L.G. Althaus, A.M. Serenelli, J.A. Panei, A.H. Córsico, E. Garcia-Berro and C.G. Scoccola, The Formation and evolution of hydrogen-deficient post-AGB white dwarfs: The Emerging chemical profile and the expectations for the PG1159-DB-DQ evolutionary connection, Astron. Astrophys. 435 (2005) 631 [astro-ph/0502005] [INSPIRE].

[56] A.H. Córsico and L.G. Althaus, Asteroseismic inferences on gw vir variable stars in the frame of new pg 1159 evolutionary models, Astron. Astrophys. 454 (2006) 863 [astro-ph/0603736] [INSPIRE].

[57] A.H. Córsico, L.G. Althaus, O.G. Benvenuto and A.M. Serenelli, The mode trapping properties of full da white dwarf evolutionary models, Astron. Astrophys. 387 (2002) 531 [astro-ph/0203453] [INSPIRE].

[58] S.D. Kawaler, D.E. Winget and C.J. Hansen, Evolution of the pulsation properties of hot pre-white dwarf stars, Astrophys. J. 295 (1985) 547. 
[59] L.G. Althaus et al., New chemical profiles for the asteroseismology of ZZ Ceti stars, Astrophys. J. 717 (2010) 897 [arXiv: 1005.2612] [INSPIRE].

[60] C. Angulo et al., A compilation of charged-particle induced thermonuclear reaction rates, Nucl. Phys. A 656 (1999) 3 [INSPIRE].

[61] R. Kunz et al., Astrophysical Reaction Rate of ${ }^{12} C(\alpha, \gamma){ }^{16} O$, Astrophys. J. 567 (2002) 643.

[62] T. Battich et al., First axion bounds from a pulsating helium-rich white dwarf star, in preparation.

[63] J. Isern, E. García-Berro, L.G. Althaus and A.H. Córsico, Axions and the pulsation periods of variable white dwarfs revisited, Astron. Astrophys. 512 (2010) A86 [arXiv:1001.5248] [INSPIRE].

[64] M. Redaelli et al., The pulsations of PG 1351+489, Mon. Not. Roy. Astron. Soc. 415 (2011) 1220. 